\title{
Highly Sensitive Acoustic Emission Sensor Based on Polarization-maintaining and Absorption-reducing Polarization-maintaining Fiber
}

\author{
Xuefeng Li, ${ }^{1}$ Yin Zhang, ${ }^{1}$ Hexin Cao, ${ }^{1}$ Xinyu Zhang, ${ }^{2}$ and Runjie Shen ${ }^{1 *}$ \\ ${ }^{1}$ College of Electronic and Information Engineering, Tongji University, \\ 4800 Cao'an Highway, Jiading District, Shanghai 201804, P. R. China \\ ${ }^{2}$ China Datang Corporation Renewable Power Co., Limited, \\ No. 1 Caishikou Street, Xicheng District, Beijing 100053, P. R. China
}

(Received August 1, 2017; accepted December 6, 2017)

Keywords: gas-insulated switchgear, partial discharge, acoustic emission sensor, PANDA polarizationmaintaining fiber

Partial discharge (PD) usually occurs before the insulation failure of a gas-insulated switchgear (GIS). Early failures in GIS can be detected effectively by monitoring the vibration signal initiated by PD. In this paper, a new type of acoustic-emission (AE) sensor based on a polarization-maintaining and absorption-reducing (PANDA) polarization-maintaining fiber (PMF) is proposed to detect the PD-induced ultrasound information accurately in GIS. Pressure load affects the birefringence of the PMF, leading to variation in light output intensity. Simulation and experimental results indicate that the effective refractive index difference between the $x$ and $y$ polarization modes has a linear relationship with the external pressure. Therefore, pressure variations generated by ultrasound can be obtained by interference demodulation between the $x$ and $y$ polarization modes. The proposed sensor has many advantages such as simple structure, small volume, and strong environmental tolerance. The results of this work provide a new direction for more effective PD detection methods and fault diagnosis approaches.

\section{Introduction}

The gas-insulated switchgear (GIS) is widely used in extra-high-voltage (EHV) transmission and distribution systems for its useful attributes such as compact structure, high reliability, small electromagnetic pollution, and low maintenance requirements. ${ }^{(1-4)}$ The large-area power failures caused by GIS faults have caused much inconvenience and serious economic losses. ${ }^{(5-7)}$ Being one of the important performance characteristics of the GIS, the reliability of its insulation is of great importance to the safety of power systems. As insulation failure usually starts with PD activity, which is usually accompanied by secondary phenomena such as sound, light, electromagnetic waves, temperature changes, and chemical reactions, ${ }^{(8-11)}$ hidden 
internal dangers can be detected by monitoring PD so that the reliability of the equipment can be ensured.

Acoustic-emission (AE) detection is a kind of nondestructive detection technology that can reliably detect the surface discharge of insulating materials and the damage of material structures. ${ }^{(12-15)}$ At present, the commonly used AE sensors are mainly piezoelectric sensors. However, these traditional sensors cannot maintain accuracy and stability for long periods in harsh environments with high temperatures or strong electromagnetic environments, so they are unable to satisfy the requirements of on-line PD monitoring applications in a high-voltage insulator. ${ }^{(16,17)}$ Therefore, there is an urgent need for a new type of AE sensor, which would be made from a new material or by a new method, that has high stability and high sensitivity for long-period detection under extreme environmental conditions.

Fortunately, optical fiber sensors have many advantages, such as small volume, immunity to corrosion and electromagnetic interference, and tolerance to high temperatures, and have been widely used in industry. ${ }^{(18-21)}$ Among these kinds of optical fiber sensors, the most widely researched in recent years are the phase modulation sensors, including Fabry-Perot interferometers (FPIs), Mach-Zehnder interferometers (MZIs), and Sagnac interferometers, ${ }^{(22-24)}$ and the wavelength modulation sensors, including the fiber Bragg grating (FBG), long-period fiber grating (LPFG), and surface plasmon resonance (SPR). ${ }^{(25-27)}$ They all have the advantages of high sensitivity and large bandwidth, but the former's response speed is not very fast, and the latter has a complex structure.

In this study, a new type of optical fiber AE sensor based on polarization modulation is proposed, analyzed, and fabricated. The sensor is equipped with a polarization-maintaining and absorption-reducing (PANDA) polarization-maintaining fiber (PMF) as the sensing unit and is used to measure the AE signals of surface discharge or material damage. First, the performance of the sensor is analyzed by finite element method (FEM) simulation in this study. The results show that the birefringence of the PMF is linearly dependent on the magnitude of the surface pressure load caused by AE. Then, an experimental platform is established and the proposed AE sensor is fabricated to test and verify the performance. The results confirm that this type of sensor has many advantages, including high sensitivity, high linear resolution, and fast response, as well as large bandwidth.

\section{Sensing Principle}

The structure of the optical fiber pressure sensor based on polarization modulation is shown in Fig. 1. The linearly polarized light is injected at $45^{\circ}$ to the fast axis ( $x$-axis) of the fiber. The $x$ and $y$ components of the linear polarization state are maintained by the high-birefringence fiber with the different propagation constants $\beta_{x}$ and $\beta_{y}$, leading to a phase difference between the two components at the output end. To detect the phase difference, the output light is separated into two perpendicularly polarized light components. The $y$ component is rotated by $90^{\circ}$ and combines with the $x$ component to form a light interferometry pattern.

The output interference light intensity is expressed by Eq. (1), where $L$ is the length of fiber, $n_{x}$ and $n_{y}$ are the effective refractive indices of $x$ - and $y$-polarized light, respectively, $k_{0}=2 \pi / \lambda$ is 


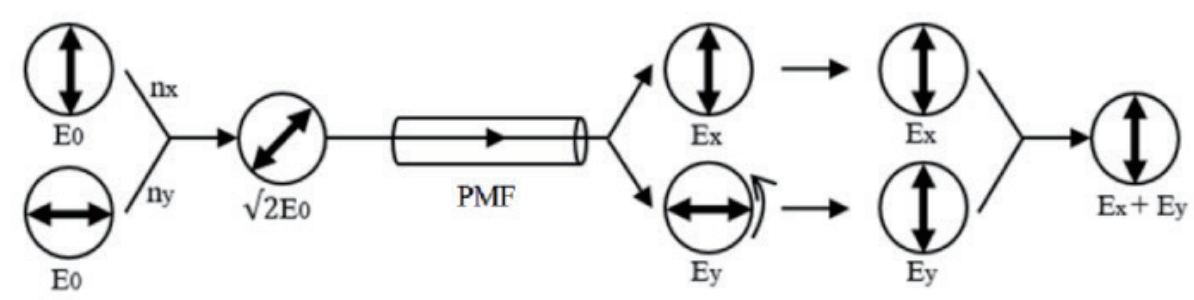

Fig. 1. Sensing principle.

the wave number in vacuum, and the relationship between the effective refractive index and the propagation constant is $\beta=k_{0} n_{\text {eff }}$.

$$
\begin{aligned}
& I=\left|E_{x}+E_{y}\right|^{2}=E_{0}^{2} \cdot\left|e^{-j \Phi_{1}}+e^{-j \Phi_{2}}\right|^{2}=2 E_{0}^{2} \cdot\left[1+\cos \left(k_{0} L B\right)\right] \\
& \Phi_{1}=\frac{2 \pi L \cdot n_{x}}{\lambda}=k_{0} n_{x} L, \Phi_{2}=\frac{2 \pi L \cdot n_{y}}{\lambda}=k_{0} n_{y} L
\end{aligned}
$$

$B=\left|n_{x}-n_{y}\right|=k_{0}\left|\beta_{x}-\beta_{y}\right|$, the birefringent coefficient, is the key factor in the output interference light intensity. When the fiber is under external pressure, the optical properties change owing to the photoelastic effect, ${ }^{(28)}$ resulting in the change of birefringent coefficient $B$, and consequently affecting the output light intensity.

\section{Performance Simulation}

In this study, the performance of the sensor is analyzed by the FEM. For this, COMSOL Multiphysics ${ }^{\circledR}$ is used to calculate the properties of the two orthogonal polarization states of the fiber under pressure. The SM15-PS-U25D PANDA PMF made by Japan Fujikura Ltd. is used as the sensing unit.

As shown in Fig. 2(a), the tensile stress between the stress-applying parts (SAPs) applied to the core induces large birefringence. In this work, we are aiming to investigate the stress distribution and the mode effective refractive index on the section of fiber under the influence of an AE signal, so the 2-D space dimension is selected to build the cross-sectional model of the PMF. The distribution of stress in the components under a zero pressure load is shown in Fig. 2(b). The stress direction is along the slow axis, pointing toward the interior of the fiber. Stress distributions in the fast axis direction and the slow axis direction under different pressure loads from 0 to $100 \mathrm{MPa}$ are shown in Figs. 2(c) and 2(d). There is a good linear relationship between stress distribution and uniform pressure load.

The birefringent properties of PANDA PMF are presented in Figs. 3(a) and 3(b). It can be inferred that an external pressure load contributes to an increase in both $x$ and $y$ polarization modes, with linear relationships of $5.51 \times 10^{-6}$ and $2.50 \times 10^{-6} \mathrm{RIU} / \mathrm{MPa}$, respectively. However, the different rates of their increases weaken the birefringence caused by geometric asymmetry. In other words, the birefringence decreases linearly with the external pressure load. Owing to its good mechanical sensitivity, the birefringence changes with external pressure load and decreases by $52.8 \%$ at $100 \mathrm{MPa}$ with a linear relationship of $3.01 \times 10^{-6} \mathrm{RIU} / \mathrm{MPa}$. 


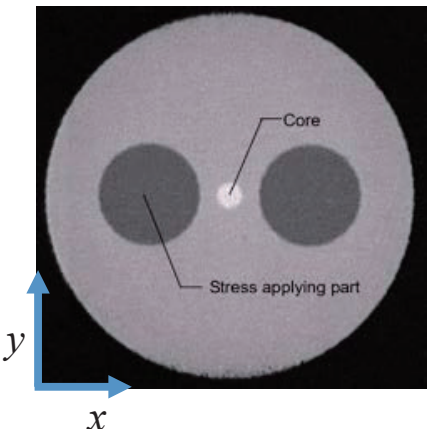

(a)

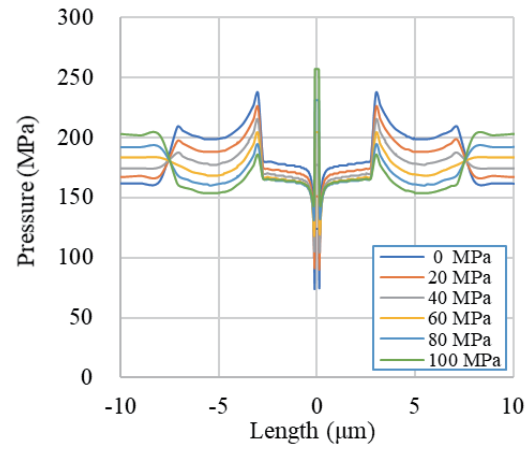

(c)

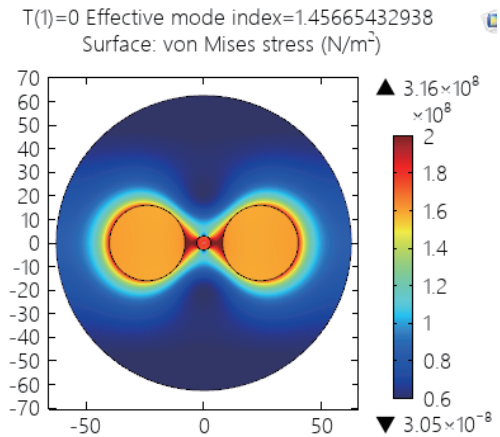

(b)

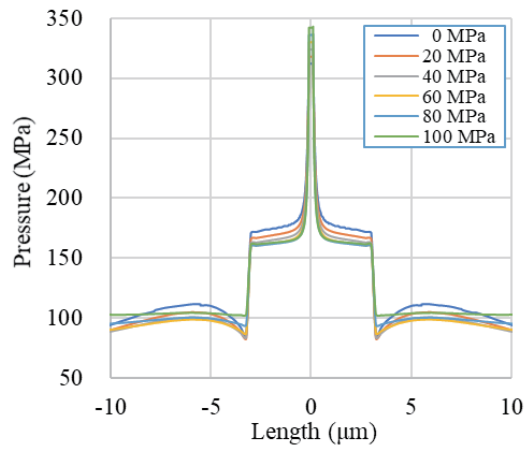

(d)

Fig. 2. (Color online) (a) Cross-sectional image of PANDA PMF, (b) stress distribution in cross section of PMF, (c) stress distribution in the slow $(x)$ axis direction with external pressure loads from 0 to $100 \mathrm{MPa}$, and (d) stress distribution in the fast $(y)$ axis direction with external pressure loads from 0 to $100 \mathrm{MPa}$.

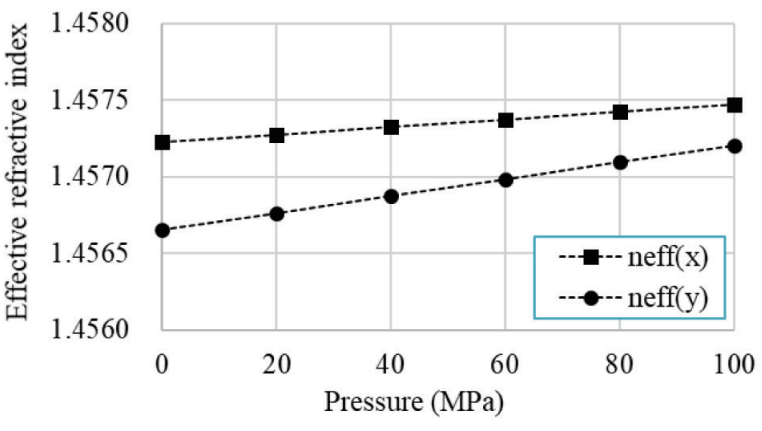

(a)

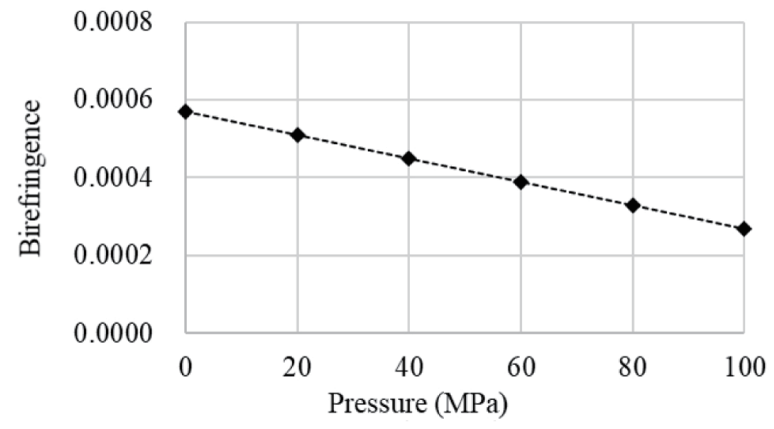

(b)

Fig. 3. Polarization modes with pressure loads from 0 to $100 \mathrm{MPa}$ along slow axis. (a) Relationship between effective refractive index and pressure and (b) relationship between birefringence and pressure.

\section{Experiment and Analysis}

In accordance with the sensing model based on polarization modulation shown in Fig. 1, the experimental platform is designed to be as shown in Fig. 4(a). The structure of the platform includes a light source (1), linear polarization controller (2), sensing unit (3), fiberbased polarization beam splitters (4), fiber-based beam coupler (5), photoelectric detector (6), 


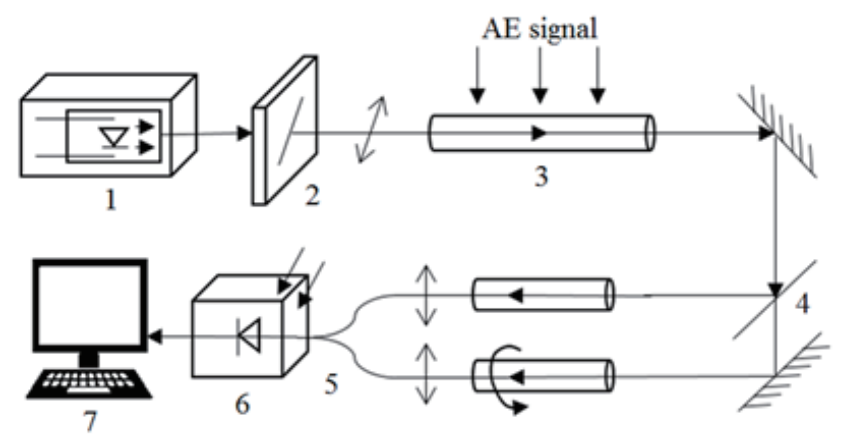

(a)

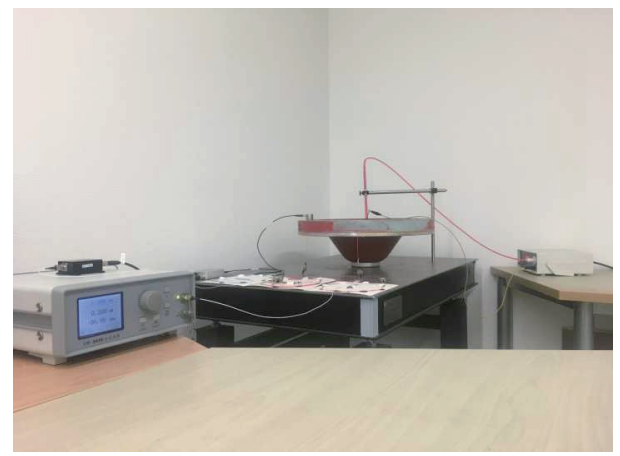

(b)

Fig. 4. (Color online) Measuring system. (a) Structure of experimental platform and (b) pot insulator containing the packaged optical fiber sensor.

and terminal for data acquisition and processing (7). As shown in Fig. 4(b), the sensing unit of the optical fiber sensor is packaged in the U-slot of a pot insulator using epoxy resin with high chemical stability and adjustable elastic parameters. The entire package is an independent assembly structure. In addition, the PXR03 piezoelectric sensor made by China PengXiang Ltd. is used for comparison. It has a wide bandwidth of $20-110 \mathrm{kHz}$.

The light source provides a stable beam of $1550 \mathrm{~nm}$ wavelength. The linear polarization controller is used to convert the unpolarized light emitted by the laser to linearly polarized light and to rotate the linearly polarized light from the slow axis to $45^{\circ}$ before it is injected to the sensing unit. The fiber-based polarization beam splitter is used to split the light from the sensing unit into two beams with orthogonally polarized directions. The fiber-based beam coupler combines two beams into one by tapering technology. When the photoelectric detector unit transmits the signal to the data acquisition card, the collected data will be transmitted to the PC terminal and stored.

\subsection{Frequency characteristics}

The sensor can theoretically detect ultrasonic signals in the broadband frequency range. In order to obtain the working frequency bandwidth of the fiber AE sensor, a passive buzzer with an adjustable vibration frequency is used as an AE source to evaluate the performance of the sensor. The signal generator outputs a sine wave voltage signal to energize the buzzer. The excitation signal frequencies are $1,10,50$, and $100 \mathrm{kHz}$.

The time domain signal is shown in Fig. 5(a). The voltage drifts greatly from $0-1.2 \mathrm{~V}$, presenting high DC and low-frequency components. This is mainly because the fiber AE sensor is easily affected by temperature, which results in polarization phase interference. In addition, the intensities under different excitation frequencies are slightly different, which is mainly caused by the passive buzzer, because, at different frequencies, the buzzer output performance is not balanced and varies from $0-2 \pi$. Through fast Fourier transform (FFT), the frequency domain signal is obtained and shown in Fig. 5(b). The high-frequency component of the signal provides phase interference information caused by the buzzer signal affecting the sensor. As 


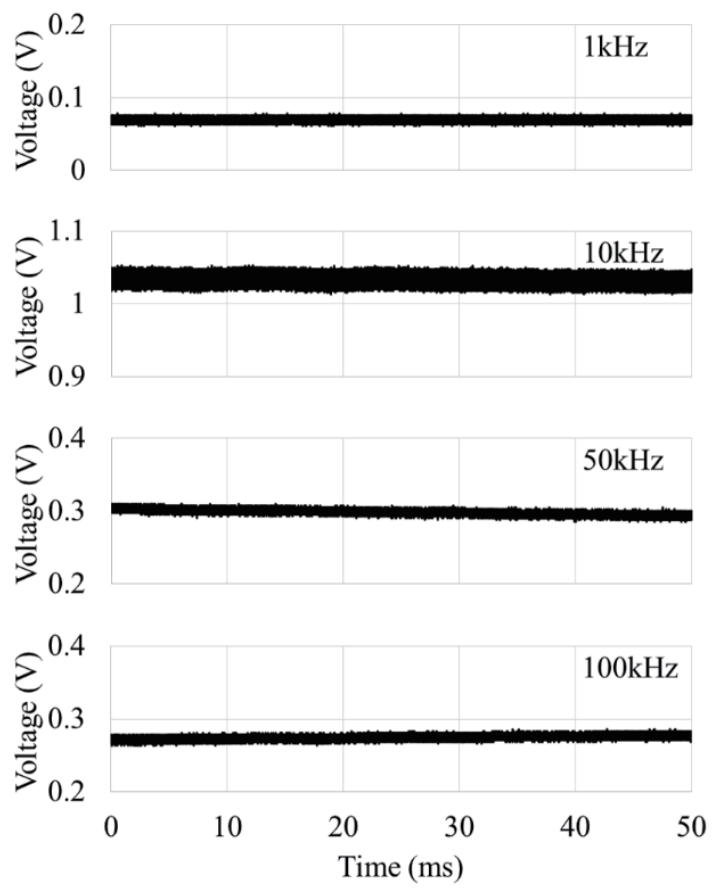

(a)

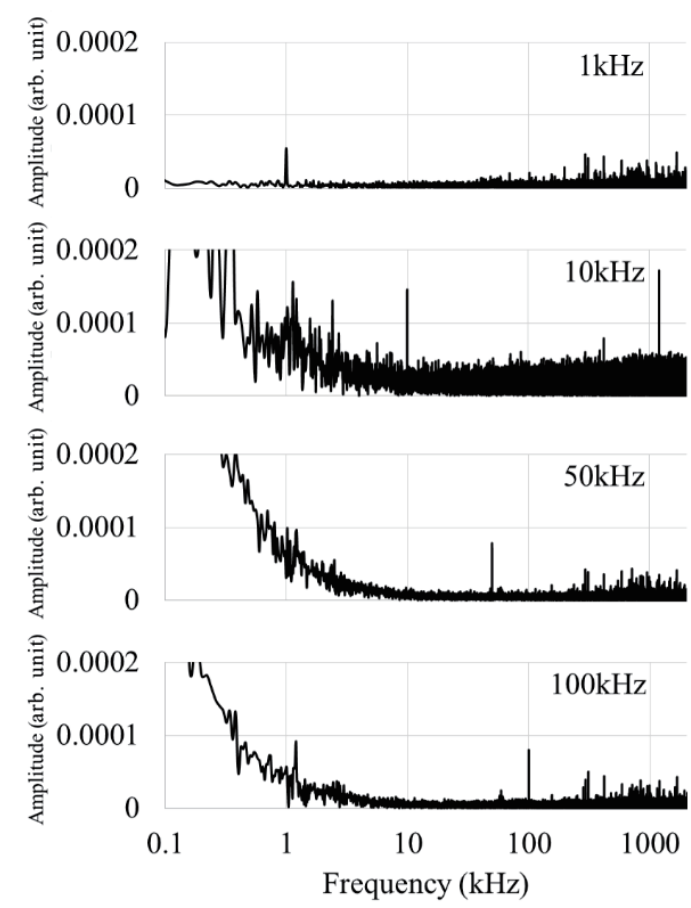

(b)

Fig. 5. Dynamic frequency response test of the optical fiber sensor. (a) AE signals in time domain and (b) AE signals in frequency domain.

the temperature mostly influences low-frequency information, it can be easily removed with a band-pass filter, and there is no interference for the detection of the ultrasonic signal.

\subsection{PD detection}

In order to confirm that the polarization-mode interferometric fiber optic AE sensor can effectively collect the weak ultrasonic signals produced by GIS PD, the performance of the sensor was evaluated using the needle-plate discharge model. The high-voltage electrode was a tungsten alloy discharge pin. The discharge spacing was $10 \mathrm{~mm}$. The discharge voltage was 40 $\mathrm{kV}$.

AE signals obtained by both sensors are shown in Fig. 6. The AE signal received by the piezoelectric sensor exhibits good waveform characteristics. There is an apparent voltage increase at the arrival of the ultrasonic signal, and then it exponentially decays to the background noise level, as shown in Fig. 6(a). In contrast, the fiber optic sensor receives a large amount of background noise and the PD-induced ultrasonic signal is not obvious, as shown in Fig. 6(b). Upon bandpass filtering from 20 to $120 \mathrm{kHz}$, the AE signal of the optical fiber sensor is much clearer than the AE signal of the piezoelectric sensor, as shown in Figs. 6(c) and 6(d). This is because low-frequency components and high-frequency components of signals have been filtered to reduce noise. Results showed that the proposed sensor showed a higher signalto-noise ratio. 


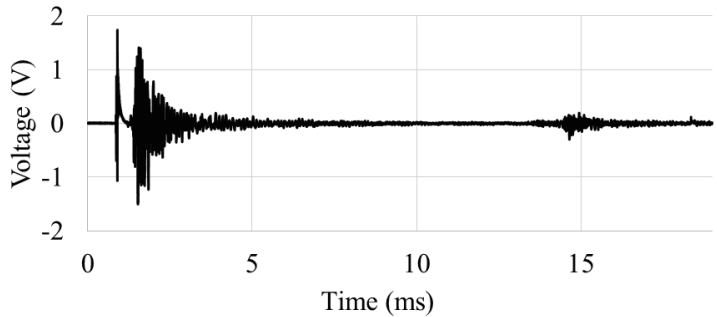

(a)

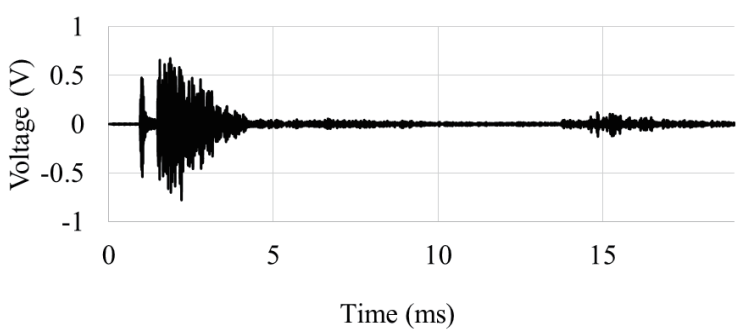

(c)

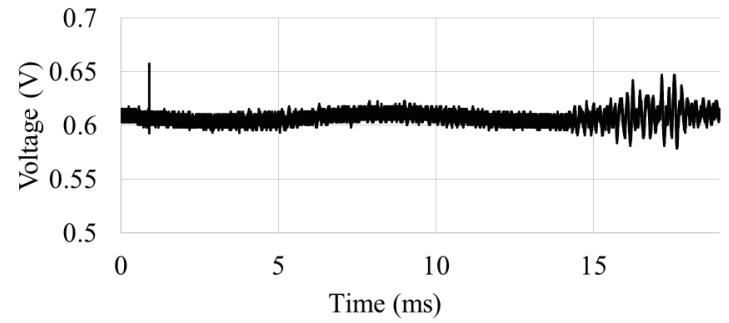

(b)

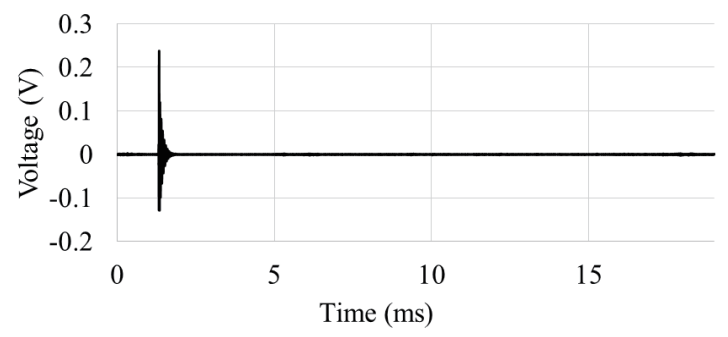

(d)

Fig. 6. Response test of AE signals generated by PD phenomenon. (a) The signal from piezoelectric sensor, (b) the signal from optical fiber sensors, (c) signal in (a) after bandpass filtering from 20 to $120 \mathrm{kHz}$, and (d) signal in (b) after bandpass filtering from 20 to $120 \mathrm{kHz}$.

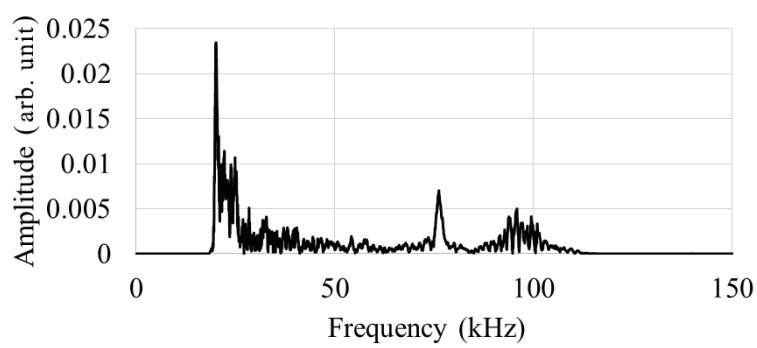

(a)

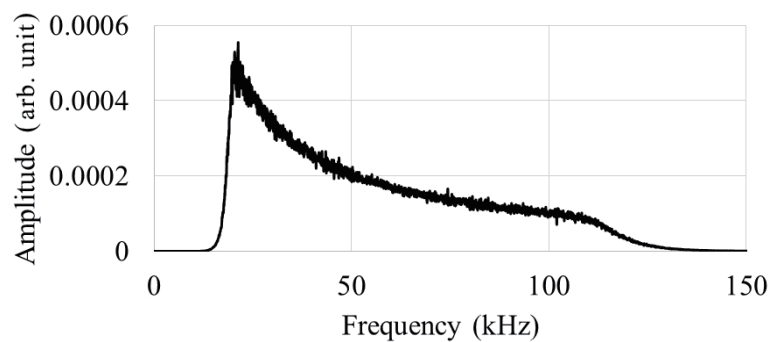

(b)

Fig. 7. AE signals in the frequency domain initiated by PD, where low-frequency components and high-frequency components have been filtered. (a) The signal from the piezoelectric sensor and (b) the signal from the optical fiber sensor.

Spectra of two AE signals are shown in Fig. 7. The piezoelectric sensor signal has a significant peak at $20 \mathrm{kHz}$. The frequency range between approximately 80 and $100 \mathrm{kHz}$ also has some smaller peaks. The fiber optic sensor has a power distribution in the bandwidth range of $20-110 \mathrm{kHz}$ with a peak between $20-30 \mathrm{kHz}$ and a decrease in power as the frequency increases. The results show that a narrow-band bandpass filter can be used in industrial inspection to further improve the signal-to-noise ratio.

\section{Conclusions}

In this study, an optical fiber AE sensor based on polarization mode interference was proposed and fabricated, and the theoretical analysis and experimental verification were carried 
out. A GIS PD detection platform based on the needle-plate discharge model was set up. The characteristic peak of the induced ultrasonic signal was between $20-30 \mathrm{kHz}$. Theoretical and experimental data were basically consistent, and the GIS PD fault was effectively monitored. The sensor is highly versatile and extensible, and it can be applied to real-time monitoring in almost any field, such as crack detection in nuclear power equipment, PD monitoring in highvoltage insulators, and material performance and fatigue testing in large buildings or aerospace components, as well as geological and seismic detection.

\section{Acknowledgments}

This work is partially supported through Science and Technology Project (Nos. 10-12) of China Datang Corporation Renewable Power Co., Ltd.

\section{References}

1 R. Benato, P. Brunello, and L. Fellin: IEEE Trans. Power Delivery 25 (2010) 2717.

2 P. Ponchon, M. Bues, H. Bosia, and G. F. Montillet: IEEE Trans. Power Delivery 21 (2006) 1935.

3 Y. B. Shu, W. J. Chen, Z. B. Li, M. Dai, C. G. Li, W. D. Liu, and X. L. Yan: IEEE Trans. Power Delivery 28 (2013) 458.

4 H. J. Ju, K. C. Ko, and D. K. Kim: IEEE Trans. Dielectr. Electr. Insul. 20 (2013) 1749.

5 L. P. Li, J. Tang, and Y. L. Liu: J. Electr. Eng. Technol. 20 (2015) 1765.

6 H. Shinkai, H. Goshima, and M. Yashima: Electr. Eng. 176 (2011) 22.

7 T. Tsuboi, G. Ueta, and S. Okabe: IEEE Trans. Dielectr. Electr. Insul. 21 (2014) 988.

8 R. Bell, C. Charlson, S. Halliday, T. Irwin, J. Lopez-Roldan, and J. Nixon: IEEE Trans. Power Delivery 18 (2003) 1187.

9 M. Szewczyk, J. Pawłowski, K. Kutorasiński, W. Piasecki, M. Florkowski, and U. Straumann: IEEE Trans. Power Delivery 30 (2015) 2331.

10 L. P. Li, J. Tang, and Y. L. Liu: IEEE Trans. Dielectr. Electr. Insul. 22 (2015) 1080.

11 M. Beltle, A. Muller, and S. Tenbohlen: IEEE Electr. Insul. Mag. 28 (2012) 17.

12 L. N. Stepanova and E. S. Tenitilov: Russ. J. Nondestruct. 48 (2012) 662.

13 F. Qiu, G. Dai, Y. Zhang, Y. T. Zhao, and C. Z. Li: Petrol. Explor. Dev. 9 (2015) 54.

14 P. Kundu, N. K. Kishore, and A. K. Sinha: Appl. Acoust. 73 (2012) 395.

15 E. Grossmann and K. Feser: IEEE Trans. Power Delivery 20 (2005) 158.

16 C. Boya, M. Ruiz-Llata, J. Posada, and J. A. Garcia-Souto: IEEE Trans. Dielectr. Electr. Insul. 22 (2015) 1663.

17 Q. Xie, S. Y. Cheng, F. C. Lü, and Y. Q. Li: Meas. Sci. Technol. 25 (2014) 35102.

18 G. Wild and S. Hinckley: IEEE Sens. J. 8 (2008) 1184.

19 S. Villalba and J. R. Casas: Mech. Syst. Sig. Process. 39 (2013) 441.

20 Y. Zhao: IEEE Sens. J. 3 (2007) 335

21 C. K. Y. Leung, K. T. Wan, D. Inaudi, X. Y. Bao, W. Habel, Z. Zhou, J. P. Ou, M. Ghandehari, H. C. Wu, and M. Imai: Mater. Struct. 48 (2015) 71.

22 S. Liu, Y. Wang, C. Liao, G. Wang., Z. Li, Q. Wang, J. Zhou, K. Yang, X. Zhong, J. Zhao, and J. Tang: Opt. Lett. 39 (2014) 2121.

23 B. Sun, Y. J. Huang, and Y. P. Wang: Opt. Express 23 (2015) 14596.

24 C. Kaczmarek and W. Wójcik: Opt. Appl. 45 (2015) 5.

25 Q. Zhang, N. Liu, T. Fink, H. Li, W. Peng, and M. Han: IEEE Photon. Technol. Lett. 24 (2012) 1519.

26 X. Zhong, Y. Wang, C. Liao, S. Liu, J. Tang, and Q. Wang: Opt. Lett. 40 (2015) 1791

27 D. P. Duarte, N. Alberto, L. Bilro, and R. Nogueira: J. Lightwave Technol. 33 (2015) 4606.

28 S. Revathi, S. R. Inabathini, and J. Pal: OPTIK 126 (2015) 3395. 


\section{About the Authors}

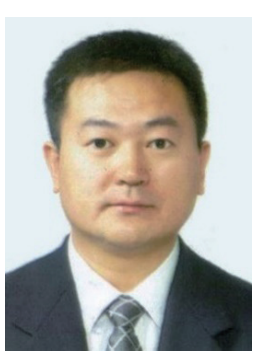

Xuefeng Li graduated from Shenyang Institute of Engineering, China, in 1999. He received his M.E. and D.E. degrees from Fukuoka Institute of Technology, Fukuoka, Japan, in 2004 and 2007, respectively. From 2007 to 2013, he was a postdoctorate and assistant researcher at Waseda University, Kitakyushu, Japan. Since 2010, he has been an associate professor at Tongji University. His research interests are in sensors and non-destructive testing (NDT).

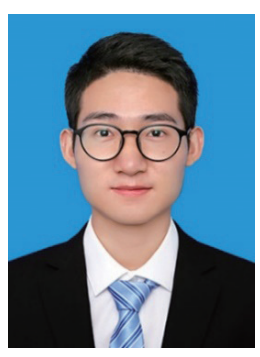

Yin Zhang received his B.S. degree from Tongji University, China. Since 2015, he has been studying at Tongji University for his M.S. degree. His research interests are in sensors and NDT.

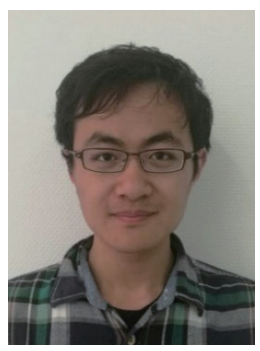

Hexin Cao received his B.S. degree from HeFei University of Technology, China, in 2016. Since 2016, he has been studying at Tongji University for his M.S. degree. His research interests are in sensors and NDT.

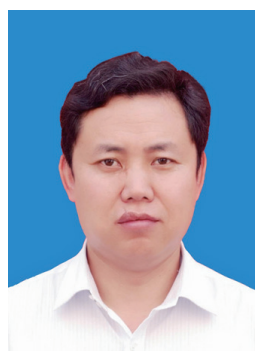

Xinyu Zhang received his B.S. degree from Northeast Electric Power University, China, in 1997. Since 2016, he has been the chief engineer at East China branch of China Datang Corporation Renewable Power Co., Ltd.

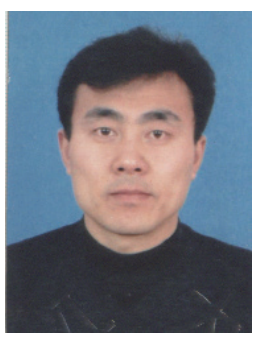

Runjie Shen received his B.S. degree from Xi'an Polytechnic University, China, in 1995. His M.S. degree was received from Xi'an Jiaotong University, China, in 1998 and his Ph.D. degree from Zhejiang University, China, in 2004. From 2004 to 2006, he was a postdoctorate at Zhejiang University. In 2006, he became an associate researcher at Zhejiang University. Since 2011, he has been an associate researcher at Tongji University. His research interests are in sensors and NDT. 